\title{
Elastic analysis of the energy and relaxation of stepped surfaces
}

\author{
D.J. Srolovitz ${ }^{a}$ and J.P. Hirth ${ }^{b}$ \\ "Department of Materials Science and Engineering, University of Michigan, Ann Arbor, MI 48109-2136, USA \\ ${ }^{b}$ Department of Mechanical and Materials Engineering. Washington State University. Pullman, WA 99164-2920, USA
}

Received 27 September 1990; accepted for publication 29 January 1991

\begin{abstract}
We present an analytical, elastic analysis for the energy and relaxation of stepped surfaces. The analysis is based upon the observation that the most prominent feature of the non-reconstructive surface relaxation consists of the atoms at the top of the ledges relaxing inwards toward the bulk. This is modeled by replacing the true atomic structure with a continuum elastic half space subjected to a periodic array of line forces (with the periodicity of the steps) directed normal to the free surface. This model is then employed to determine the stress, strain and displacement fields and elastic energy associated with the surface relaxation. We find that the stress and strain fields decay quickly into the bulk as $Y \mathrm{e}^{-Y}$, where $Y$ is the distance from the surface normalized by the interledge spacing. The surface energy is largely controlled by the terrace energy and the ledge energy, while the ledge interaction energy decays as the inverse square of the ledge spacing. The elastic model provides an accurate description of the wavelength, phase and decay rate of the surface relaxations compared with atomistic simulation results for metals.
\end{abstract}

\section{Introduction}

Over the last decade, low-energy electron diffraction studies [1] (LEED) and ion scattering experiments [2] at a variety of energies have converged to provide a coherent picture of surface relaxation (i.e., the non-reconstructive changes in the positions of atoms on a surface relative to their positions in a bulk perfect crystal). For stepped metallic surfaces, these studies have shown that the most pronounced feature of surface relaxation is the movement of the outermost atoms inward toward the bulk crystal. This inward relaxation of these atoms may be attributed to the adjustment of the positions of the atoms to restore the electron density deficit associated with the creation of the free surface [3]. Clearly, this electron density deficit is largest near the lowest coordinated atom. The change in the interlayer spacings parallel to the free surface, relative to their perfect crystal value, either decays monotonically towards the bulk spacing or show an oscillatory relaxation. Atomistic simulations using interatomic potentials designed for metals show similar surface relaxations [4]. While the magnitude of the inward relaxations of the outermost atoms depends sensitively on the details of the atomic interactions, we believe that the nature of the atomic relaxations of underlying atomic planes is primarily a consequence of the large inward relaxation of the outermost atoms. Consequently, we present an analysis of surface relaxation and surface energy based entirely on the elastic consequences of the inward relaxation of the outermost atoms.

In our analysis, we replace the atomic picture of surface ledges and terraces with a continuum elastic picture of a linear elastic half-space that is subject to a periodic array of parallel line forces directed inward toward the bulk crystal. This periodic array of parallel line forces plays the role of the relaxed surface ledges. The data needed to parameterize the present theory are simply the elastic properties of the bulk crystals and the surface crystallography. The idea of replacing the actual ledge distribution with line forces was first alluded to by Marchenko and Parshin [5] to describe the interaction between ledges. Chen, Voter 
and Srolovitz [4] employed line forces in a preliminary description of surface relaxation. In the present paper, we present a more complete elastic analysis of these surface relaxations and surface energies and compare our predictions with simulation results. Lau and Kohn [6] employed a similar approach to analyzing the interactions between atoms adsorbed on a solid surface. In a similar vein, Marchenko [7,8] and Alerhand et al. [9] used an elastic analysis of the forces produced at the interface between different surface structures (e.g., reconstructions) to analyze surface phase transitions and external stress effects. In most of the earlier analyses, the interactions were developed for the case of pairs of forces.

The full elastic fields associated with a stepped surface, as analyzed in terms of a periodic array of line forces, are developed in the following section. These results are then applied to the determination of the energy of a stepped surface and to surface relaxation in sections 3 and 4 , respectively. Before making detailed comparisons of these predictions with simulation results, we present an analysis of the crystallography of stepped surfaces, with particular attention paid to surfaces with (100) and (111) terraces on crystals with cubic symmetry. Finally, the predicted miscut angle dependence of the energies of stepped surfaces is compared with atomistic simulation results. The predicted surface relaxation profiles are also compared with those from atomistic simulations [10].

\section{Elastic solution}

As discussed above, the most prominent feature of the displacement field associated with the relaxation of stepped surfaces is that the outermost atoms (i.e., those on the top of the ledges) have the largest inward relaxation. In the present analysis, we do not attempt to determine the magnitude of the relaxation of the outermost atoms, but instead focus on the elastic consequences of their inward relaxation. These results will then be employed to determine the energy of stepped surfaces and many well-known features of surface relaxation.

We begin by considering a surface made up of a periodic array of ledges and terraces. We model

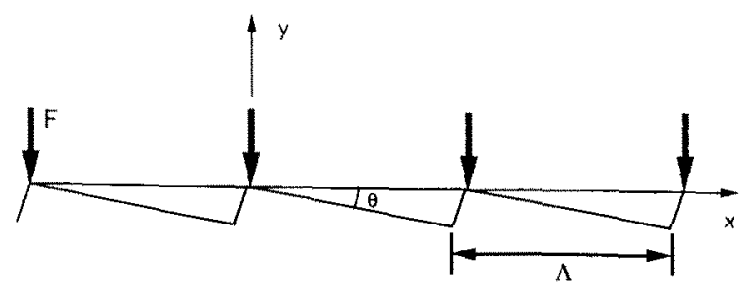

Fig. 1. The surface is modeled as a continuous elastic half space with forces $F$ directed inward, normal to the vicinal surface. The force spacing $\Lambda$ is equal to the interledge spacing and the angle between the terraces and the vicinal surface is $\theta$.

the inward motion of the atoms along the ledges with lines of force that act normal to the nominal surface, as shown in fig. 1. A single line of force acting on a flat surface bounding an elastic half space results in the following stress distribution [11] in the solid:

$$
\begin{aligned}
& \sigma_{x x}=-\frac{2 F}{\pi} \frac{x^{2} y}{r^{4}}, \\
& \sigma_{y y}=-\frac{2 F}{\pi} \frac{y^{3}}{r^{4}}, \\
& \sigma_{x y}=-\frac{2 F}{\pi} \frac{x y^{2}}{r^{4}}, \\
& \sigma_{z z}=-\frac{2 \nu F}{\pi} \frac{y}{r^{2}}, \\
& \sigma_{x z}=\sigma_{y z}=0,
\end{aligned}
$$

where the coordinate system is defined in fig. $1, F$ is the magnitude of the applied force per unit length, $r=\left(x^{2}+y^{2}\right)^{1 / 2}$, and $\nu$ is the Poisson ratio of the elastically isotropic solid. The associated strain field can be obtained from Hooke's law:

$\epsilon_{x x}=\frac{F}{\mu \pi} \frac{y}{r^{2}}\left(\nu-\frac{x^{2}}{r^{2}}\right)$,

$\epsilon_{y y}=\frac{F}{\mu \pi} \frac{y}{r^{2}}\left(p-\frac{y^{2}}{r^{2}}\right)$,

$\epsilon_{x y}=-\frac{2 F}{\mu \pi} \frac{x y^{2}}{r^{4}}$.

$\epsilon_{z z}, \epsilon_{x z}$ and $\epsilon_{y z}$ are all zero because this is a plane strain problem. The displacement field associated 
with the line force is found via a straightforward integration of eq. (2):

$u_{x}=\frac{F}{2 \mu \pi}\left[\frac{x y}{r^{2}}-(1-2 \nu) \tan ^{-1}\left(\frac{x}{y}\right)\right]$

$u_{y}=\frac{F}{2 \mu \pi}\left[\frac{-x^{2}}{r^{2}}-2(1-\nu) \ln \left(\frac{r}{b}\right)\right]$,

where $b$ is a cut-off distance, smaller than which linear elastic continuum theory no longer applies ( $b$ is generally taken to be of the order of a lattice parameter).

In order to obtain the elastic field of the periodically stepped surface, we sum the above stress, strain and displacements fields over an infinite number of regularly spaced parallel line forces, i.e.,

$\rho_{\alpha \beta}(x, y)=\sum_{i=-\infty}^{\infty} \rho_{\alpha \beta}^{0}(x+i \Lambda, y)$,

where $\rho_{\alpha \beta}$ is a tensor field corresponding to a uniform parallel array of line forces spaced a distance $\Lambda$ apart and $\rho_{\alpha \beta}^{0}$ is the value of that tensor field for a single line force. The details of how the resultant sums are solved for the particular cases of interest here are discussed in the appendix. Since an array of line forces directed into the material would result in an overall compression in the material, the far-field stresses, strains and displacements are subtracted off those found by summing eqs. (1)-(3). This guarantees that far from the surface, the material in unstrained.

Inserting eqs. (1) and (2) into eq. (4) and writing $X=2 \pi x / \Lambda, Y=2 \pi y / \Lambda$ and $R=\left(x^{2}+\right.$ $\left.y^{2}\right)^{1 / 2}$, we obtain the stresses and strains for the periodic array of line forces:

$$
\begin{aligned}
\sigma_{x x}= & -\frac{F}{\Lambda}\left[\frac{\sinh (Y)}{\cosh (Y)-\cos (X)}\right. \\
& \left.+Y \frac{1-\cosh (Y) \cos (X)}{[\cosh (Y)-\cos (X)]^{2}}-1\right]
\end{aligned}
$$

$$
\begin{aligned}
& \sigma_{y y}=-\frac{F}{\Lambda}\left[\frac{\sinh (Y)}{\cosh (Y)-\cos (X)}\right. \\
& \left.-Y \frac{1-\cosh (Y) \cos (X)}{[\cosh (Y)-\cos (X)]^{2}}-1\right] \text {, } \\
& \sigma_{x y}=-\frac{F}{\Lambda} Y \frac{\sinh (Y) \sin (X)}{[\cosh (Y)-\cos (X)]^{2}} \text {, } \\
& \epsilon_{x x}=-\frac{F}{2 \mu \Lambda} \\
& \times\left[-(1-2 \nu)\left(\frac{\sinh (Y)}{\cosh (Y)-\cos (X)}-1\right)\right. \\
& \left.-Y \frac{1-\cosh (Y) \cos (X)}{[\cosh (Y)-\cos (X)]^{2}}\right] \text {, } \\
& \epsilon_{y y}=-\frac{F}{2 \mu \Lambda} \\
& \times\left[-(1-2 \nu)\left(\frac{\sinh (Y)}{\cosh (Y)-\cos (X)}-1\right)\right. \\
& \left.+Y \frac{1-\cosh (Y) \cos (X)}{[\cosh (Y)-\cos (X)]^{2}}\right] \text {, } \\
& \epsilon_{x y}=-\frac{F}{\mu \Lambda} Y \frac{\sinh (Y) \sin (X)}{[\cosh (Y)-\cos (X)]^{2}} \text {. }
\end{aligned}
$$

For large $Y$, these reduce to:

$\sigma_{x x}=-\frac{F}{\Lambda} Y \mathrm{e}^{-Y} \cos (X)$,

$\sigma_{y y}=\frac{F}{\Lambda} Y \mathrm{e}^{-Y} \cos (X)$,

$\sigma_{x y}=-\frac{F}{\Lambda} Y \mathrm{e}^{-Y} \sin (X)$,

$\sigma_{z z}=0$,

$\epsilon_{x x}=-\frac{F}{2 \mu \Lambda} Y \mathrm{e}^{-Y} \cos (X)$,

$\epsilon_{y y}=\frac{F}{2 \mu \Lambda} Y \mathrm{e}^{-Y} \cos (X)$,

$\epsilon_{x y}=-\frac{F}{\mu \Lambda} Y \mathrm{e}^{-Y} \sin (X)$.

In all cases, the elastic fields decay exponentially into the bulk solid and have a periodic structure in the direction parallel to the free surface.

The displacement field associated with the 
parallel array of line forces may be determined by summing eqs. (3) and (4) or by integration of eq. (6). This yields

$$
\begin{aligned}
u_{x}= & \frac{F}{4 \pi \mu}\left\{Y \frac{\sin (X)}{\cosh (Y)-\cos (X)}\right. \\
& +2(1-2 \nu) \tan ^{-1}\left[\frac{\cosh (Y / 2) \sin (X / 2)}{\sinh (Y / 2) \cos (X / 2)}\right] \\
& -(1-2 \nu) X\}, \\
u_{y}= & \frac{F}{4 \pi \mu}\left\{Y \frac{\sinh (Y)}{\cosh (Y)-\cos (X)}\right. \\
& -2(1-\nu) \ln [\cosh (Y)-\cos (X)] \\
& +(1-2 \nu) Y\},
\end{aligned}
$$

where the last terms in the brackets in eqs. (9) were added to insure that the solid is stress-free far from the surface.

\section{The energy of stepped surfaces}

The energy of a stepped surface can be described in terms of a sum of three terms. The first, $\gamma_{\mathrm{T}}$, is the energy of the terrace, which may be associated with the surface energy of a flat surface with the same orientation as that of the terrace. The second term, $\gamma_{L}$ is the energy of an individual ledge associated with the additional broken bonds at a ledge compared with the flat surface. The final contribution to the energy of the stepped surface is the interaction energy between ledges, $\gamma_{\mathrm{I}}$. In this section we determine the ledge interaction energy for a uniformly stepped surface.

In order to calculate the ledge interaction energy we make use of a virtual work argument. In this method, the ledge interaction energy is determined as the work required to create a ledge on the surface in the presence of the other surface ledges. In practice, this corresponds to applying the force $F$ at the position of a ledge when that ledge is missing and all of the other ledges are present.

$\gamma_{\mathrm{I}}=F \lim _{y \rightarrow b}\left[u_{y}^{\text {ans ay }}(0, y)-u_{y}^{\text {single }}(0, y)\right]$ where the first term in the bracket is given in eq. (9b) and the second term is eq. (3b) and where, as in eq. (3b), $b$ is a cut-off distance, smaller than which linear elastic continuum theory no longer applies. Inserting eqs. (3b) and (9b) into eq. (10), we find

$$
\begin{aligned}
\gamma_{\mathrm{I}}= & \frac{F^{2}}{4 \pi \mu}\left\{B \frac{\sinh (B)}{\cosh (B)-1}\right. \\
& \left.-2(1-\nu) \ln \left[\frac{\cosh (B)-1}{B^{2}}\right]\right\},
\end{aligned}
$$

where $B=2 \pi b / \Lambda$.

In the limit that $2 \pi b \ll \Lambda$, eq. (11) reduces to

$\gamma_{\mathrm{I}}=\frac{\pi F^{2} \nu}{6 \mu}\left(\frac{b}{\Lambda}\right)^{2}$

Therefore, to lowest order, the interaction between ledges scales as the inverse square of the interledge spacing. This type of scaling was first obtained by Marchenko and Parshin [5] but with a different proportionally constant. Combining all the energy terms into a single expression for the energy per unit area of the surface and taking into account the spacing between ledges, we find

$\gamma=C_{1}+\frac{C_{2}}{\Lambda}+\frac{C_{3}}{\Lambda^{3}}$

where $C_{1}$ is $\gamma_{\mathrm{T}}$, the second accounts for the energy per ledge $\gamma_{L}$ and the final term accounts for the ledge interaction energy per unit area of the surface. In terms of the energy versus miscut angle, eq. (13) can be rewritten as

$\gamma=C_{1}+C_{2}^{\prime} \sin (\theta)+C_{3}^{\prime} \sin ^{3}(\theta)$,

where the miscut angle $\theta$ is measured as the angle between the terrace and the vicinal surface. Clearly, the numerical values of the constants in eqs. (13) and (14) must be changed when the miscut angle (ledge spacing) becomes sufficiently large (small) that the crystallography of the terraces or ledges change. Therefore, eqs. (13) and (14) should be viewed as small miscut angle approximations to the energy of stepped surfaces. Applications of eqs. (13) and (14) to particular surfaces are discussed below. 


\section{Surface relaxation}

Surface relaxation experiments are typically analyzed in terms of changes in the spacing between the atomic planes parallel to the surface [12]. Usually, only the relative displacements of the planes in the direction normal to the surface are quoted (this is the $y$-direction in fig. 1). $d_{n, n+1}$ is the separation between plane $n$ and plane $n+1$ in the $y$-direction, where plane $n=1$ corresponds to the surface plane of atoms (i.e., those that intersect the $x$-axis in fig. 1). Importantly, except in very special circumstances, atoms on adjacent planes (i.e., different $y$-coordinates) have different $x$-coordinates. This is illustrated in fig. 2 for the case of a (610) surface of a simple cubic lattice, with (100) terraces. The difference $\left(\Delta d_{n, n+1}\right)$ between $d_{n, n+1}$ and that expected for the perfect crystal $d_{0}$ can be described in terms of the displacement field calculated in section 2 , above

$$
\begin{aligned}
\Delta d_{n, n+1} & \\
= & d_{n, n+1}-a \sin (\theta)=u_{y}(n)-u_{y}(n+1) \\
= & u_{y}(n a \cos (\theta), n a \sin (\theta)) \\
& -u_{y}((n+1) a \cos (\theta),(n+1) a \sin (\theta)),
\end{aligned}
$$

where $a$ is the perfect crystal spacing between atoms on the terrace in the direction normal to the ledge and the $u_{y}$ can be obtained from eq. (9b). For large $Y, \Delta d_{n, n+1}$ behaves as $Y \mathrm{e}^{-Y} \cos (Y /$ $\tan \theta$ ) [see eq. (8b)]. This decaying oscillatory profile is reminiscent of the $\Delta d_{n, n+1}(Y)$ profile determined both experimentally [13] and by atomistic simulation [4]. A comparison between the

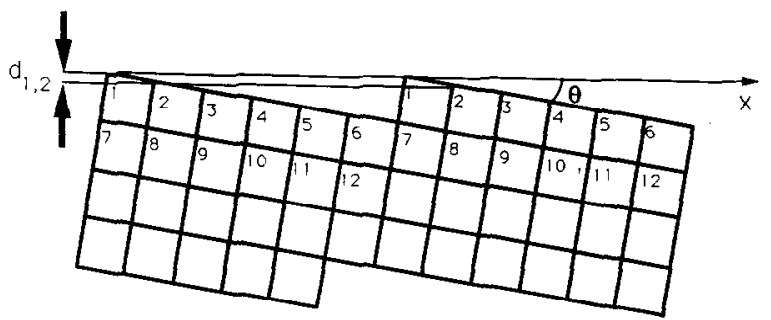

Fig. 2. The unrelaxed atomic positions for the (610) surface of a simple cubic material with (100) terraces. $d_{1,2}$ is the interplanar spacing between the surface (610) plane and the (610) plane immediately under it.

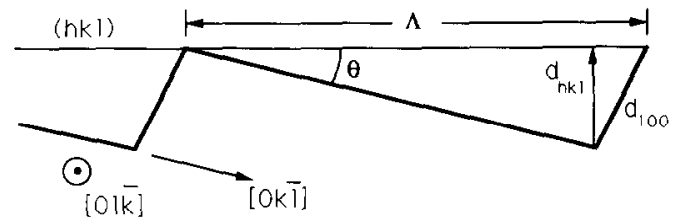

Fig. 3. The crystallography of an ( $h k l)$ surface in a cubic material.

analytical, simulation and experimental results is made below.

\section{Ledge geometry}

A prescription is now presented to relate ledge spacing to surface orientation. We first consider a vicinal surface of a face-centered cubic lattice near (100), as shown in fig. 3 . We specify the ordering of Miller indices such that $h, k$ and $l$ are all positive and $h \geqslant k \geqslant l$. From the geometry, $\cos \theta$ $=h /\left(h^{2}+k^{2}+l^{2}\right)^{1 / 2}$ and $\sin \theta=d_{100} / \Lambda$, where the interplanar spacing $d_{100}, \Lambda$, and $\theta$ are defined in fig. 3. The interplanar spacing $d_{100}$ is simply given in terms of the lattice parameter $a_{0}$ as $d_{100}=a_{0} / 2$. Combining these relations we find

$\Lambda_{h k l}=\frac{a_{0}}{2} \frac{\left(h^{2}+k^{2}+l^{2}\right)^{1 / 2}}{\left(k^{2}+l^{2}\right)^{1 / 2}}$.

Similarly, if the ledges prefer to form straight segments lying along [00피 directions separated by kinks, one can compute the kink spacing $x$ along the ledge. With the convention that $k \geqslant l$, we find

$\chi_{h k l}=\frac{a_{0}}{2} \frac{\left(k^{2}+l^{2}\right)^{1 / 2}}{k}$.

If the ledges prefer to lie along the [011] directions, the kink spacing is

$\chi_{h k l}=\frac{a_{0}}{\sqrt{2}} \frac{\left(k^{2}+l^{2}\right)^{1 / 2}}{(k-l)}$.

For vicinal surfaces near (111) with the same selection of the stereographic unit triangle, i.e., $h$, $k$ and $l$ are all positive and $h \geqslant k \geqslant l$, the ledge spacing is

$\Lambda_{h k l}=\frac{a_{0}}{\sqrt{2}} \frac{\left(h^{2}+k^{2}+l^{2}\right)^{1 / 2}}{\left(h^{2}+k^{2}+l^{2}-h k-k l-l h\right)^{1 / 2}}$. 
Al (h10)

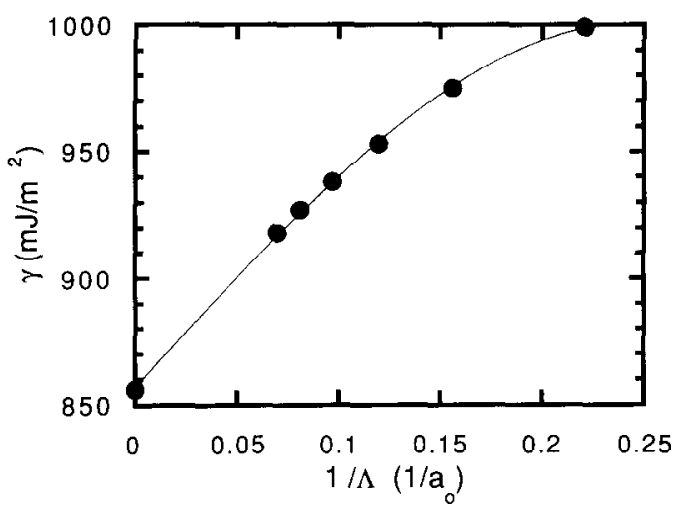

Fig. 4. The surface energy of $(h 10) \mathrm{Al}$ surfaces as a function of the inverse interledge spacing $1 / \Lambda$ (in units of the $\mathrm{Al}$ cubic lattice parameter). The solid dots represent atomistic simulation data $[10,16]$. The continuous line is the best fit of the analytical, elastic results [eq. (13)] to the simulation data.

In this case, if ledges prefer [01] directions, the kink spacing would be

$\chi_{h k l}=\frac{a_{0}}{2} \frac{\left(h^{2}+k^{2}+l^{2}-h k-k l-l h\right)^{1 / 2}}{(k-l)}$.

We note that the kinks would be geometric kinks and, as such, expected to be uniformly spaced at low temperatures. At temperatures approaching the melting point, thermal kinks would also be present, the ledges would "roughen" [14] and it would be more appropriate to think of $\Lambda$ as the mean spacing between ledges.

\section{Discussion}

To verify the theoretical predictions for the dependence of surface energy and surface structure on the spacing between ledges on stepped surfaces we compare our predictions with the atomistic simulation results of Chen et al. $[4,10]$. The atomic structure and energy of unreconstructed (100), (210), (310), (410), (510), (610) and (710) surfaces were determined using zero-temperature atomistic simulation methods and embedded atom method-like potentials for aluminium [15]. The details of the simulation method can be found in ref. [10]. Previous simulations using this type of potential have been shown to accurately predict perfect crystal properties, surface relaxation and surface reconstruction in metals $[4,15]$.

Since the predicted dependence of the surface energy on ledge spacing $\Lambda$ [eq. (13)] is a polynomial in $1 / \Lambda$, we plot the surface energy $\gamma$ versus $1 / \Lambda$ in fig. 4 . The line in fig. 4 represents the best fit of eq. (13) to the simulation data $[10,16]$ where the parameters $C_{1}, C_{2}$, and $C_{3}$ were taken as fitting parameters. The fit of eq. (13) to the simulation data is excellent (the $R$-value equals
(410)

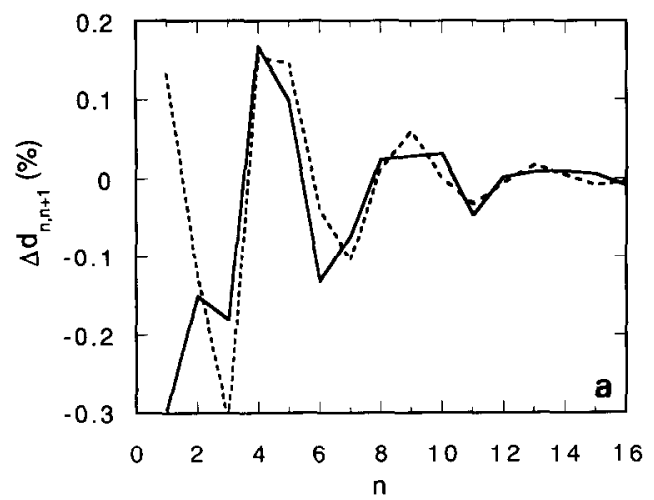

(610)

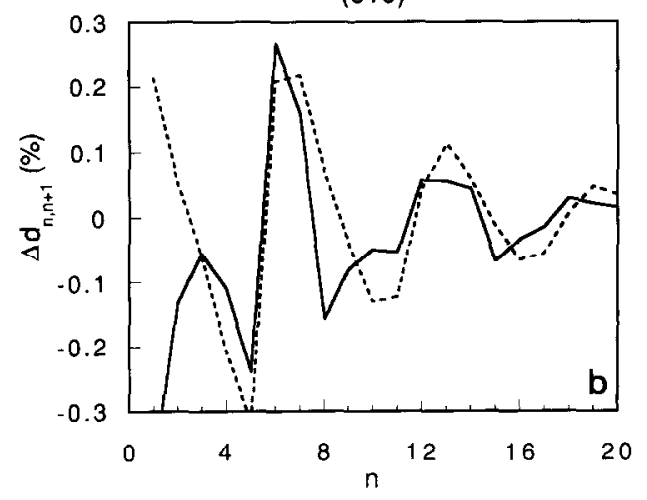

Fig. 5. Surface relaxation $\Delta d_{n, n+1}$ versus atomic layer number $n$ for the (a) (410), and (b) 610 surfaces of Al. The solid lines represent the atomistic simulation results of Chen et al. $[10,16]$ and the dotted lines represent the present analytical, elastic results [eqs. (9b) and (15)]. 
$0.99998)$. The intercept of the $\gamma$-axis at $1 / \Lambda=0$ is simply the (100) surface energy or terrace energy (for the ( $h 10)$ surfaces) and is the first term on the right hand side of eq. (13), $C_{1}=\gamma_{100}=\gamma_{\mathrm{T}}=0.856$ $\mathrm{J} / \mathrm{m}^{2}$. The slope of the curve at $1 / \Lambda=0$ yields the ledge energy per unit length or the second term on the right hand side of eq. (13), $\gamma_{\mathrm{L}}=C_{2}=0.883 \times$ $10^{-10} \mathrm{~J} / \mathrm{m}$. Finally, all the curvature in fig. 4 is associated with the ledge interaction energy per unit length $\gamma_{\mathrm{I}}=C_{3} / \Lambda^{2}=4.943 \times 10^{-30}(\mathrm{~J} \mathrm{~m}) / \Lambda^{2}$.

Both the atomistic simulations and the analytical, elastic theory derived above yield predictions for the surface relaxation $\Delta d_{n, n+1}$ as a function of depth. Figs. 5a and 5b show $\Delta d_{n, n+1}$ versus atomic layer number $n$ (measured from the outermost ( $h k l$ ) plane) for data obtained from atomistic simulations by Chen et al. $[10,16]$ and eqs. (9b) and (15) for the (410) and (610) surfaces of $\mathrm{Al}$, respectively. Since the magnitude of the force $F$ in eq. (9b) is not predicted by the analytical theory, the overall amplitudes of the theoretical curves in fig. 5 were adjusted to obtain the best fit with the simulation data. Comparison of the predictions of the analytical, elastic theory and the simulation data shows that the wavelength, phase and decay rate of the surface relaxations $\Delta d_{n, n+1}$ are in general agreement for both the (410) and (610) surfaces.

For small $n$ (i.e., within 1-3 atomic planes from the surface), the analytical theory predicts an expansion of the interlayer spacings while both simulations and experiments predict a contraction. Additionally, while we do predict the correct general shape of the relaxation curve beyond the first few atomic planes, the correspondence between the detailed shapes of the simulated and theoretically predicted relaxation curves is less than perfect. These discrepancies are attributable to our initial assumption that only the ledges are subjected to an inward force, while in reality this force should be distributed over several surface atoms. Moreover, we have assumed that the line forces applied at the ledge edges to obtain the elastic solutions were directed normal to the surface, while in reality a small component of the true force should be directed along the surface. This possibility was omitted from the present theory for the sake of simplicity. The assumptions made in the present theory will always be worst near the surface and improve with increasing depth into the crystal. If one were interested in obtaining very accurate predictions, one could employ the experimentally measured displacements of the atoms on the first few atomic planes (most experiments only measure the displacements of the first couple of atomic planes) in choosing the surface force distribution.

The scaling of the amplitude of the predicted surface relaxation curve to match the simulation results provides a measure of the magnitude of the surface line force $F$. The best fit to the simulation surface relaxation data for the (410) and (610) Al surfaces (i.e., those shown in fig. 5) was obtained with $F=2 \mathrm{~J} / \mathrm{m}^{2}$. The value for $C_{2}$ from that in fig. 4 also gives $F=2 \mathrm{~J} / \mathrm{m}^{2}$ provided the cut-off parameter $b=0.43 \mathrm{~nm}$. This cut-off distance is slightly larger than the Al perfect crystal lattice parameter. The analogous core cut-off for dislocations [17] ranges from 0.5 to 2 times the nearest neighbor spacing and the value of $b$ we obtain is well within this range. As discussed in detail for the more extensively studied dislocation case [17], the core cut-off is an artifice to account for nonlinear elastic effects in the core region. Hence the results of the surface energy analysis and the surface relaxation analysis are in very good agreement with each other and with the atomistic simulation data.

Of course, there are several possible sources of error in the present analysis. The most severe of which is the assumed surface force profile. While this force profile has the appropriate periodicity, the true distribution of surface forces are expected to be distributed rather than being point-, or line-, like. Such a continuous distribution of forces would give a more reasonable surface relaxation profile within the first few layers of the surface, where the present analysis yields erroneous results. We have also assumed in the above derivations, that the forces are directed normal to the nominal surface. We expect that the true force distribution has some components parallel to the surface. Nonetheless, despite these caveats, we have demonstrated that the basis for step interaction and multilayer relaxation is basically an elastic response to the surface forces resulting from elec- 
tronic effects at the surface. At its present level, we view the elastic theory as semi-quantitative.

\section{Conclusion}

We have presented an analytical, elastic analysis for the energy and relaxation of stepped surfaces. The analysis was based upon the observation that the most prominent feature of the non-reconstructive surface relaxation consists of the outermost atoms (i.c., those on the surfacc ledges) relaxing inwards toward the bulk. This was modeled by replacing the true atomic structure with a continuum elastic half-space subjected to a periodic array of line forces (with the periodicity of the ledges) directed normal to the free surface. This model was then employed to determine the stress, strain and displacement fields and elastic energy associated with the surface relaxation. We find that the stress and strain fields decay quickly into the bulk as $Y \mathrm{e}^{-Y}$, where $Y$ is the distance from the surface normalized by the interledge spacing. The surface energy is largely controlled by the terrace energy and the ledge energy, while the ledge interaction energy decays as the inverse square of the ledge spacing. The elastic model provides an accurate description of the wavelength, phase and decay rate of the surface relaxations compared with atomistic simulation results for metals.

\section{Acknowledgements}

We wish to thank S.P. Chen and A.F. Voter for providing unpublished surface energy and surface relaxation simulation results and $R$. Eykholt and W.H. Yang for their useful mathematical suggestions. D.J.S. work was supported by the US Air Force Office of Scientific Research through contract AFOSR-90-0112.

\section{Appendix}

The sums associated with the stress, strain and displacement fields of a uniform array of parallel line forces may be evaluated using the same types of sums employed in calculating the strain field of a low angle grain boundary from the fields of individual dislocations [17]. Starting with a result which may be found in standard texts on analysis [19],

$$
\sum_{n=-\infty}^{\infty} \frac{1}{n+a}=\pi \cot (\pi a)
$$

Adding the expressions given by eq. (A.1) for $a=p+\mathrm{i} q$ and $a=p-\mathrm{i} q$ yields

$$
\sum_{n=-\infty}^{\infty} \frac{n+p}{q^{2}+(n+p)^{2}}=\frac{\pi \sin (2 \pi p)}{\cosh (2 \pi q)-\cos (2 \pi p)}
$$

while subtraction of the same expression produces

$$
\begin{aligned}
& \sum_{n=-\infty}^{\infty} \frac{1}{q^{2}+(n+p)^{2}} \\
& =\frac{\pi}{q} \frac{\sinh (2 \pi q)}{\cosh (2 \pi q)-\cos (2 \pi p)} .
\end{aligned}
$$

Differentiating eqs. (A.2) and (A.3) with respect to $p$, one obtains, respectively

$$
\begin{aligned}
& \sum_{n=-\infty}^{\infty} \frac{q^{2}-(n+p)^{2}}{\left[q^{2}+(n+p)^{2}\right]^{2}} \\
& \quad=2 \pi^{2} \frac{\cosh (2 \pi q) \cos (2 \pi p)-1}{[\cosh (2 \pi q)-\cos (2 \pi p)]^{2}},
\end{aligned}
$$

and

$$
\begin{aligned}
& \sum_{n=-\infty}^{\infty} \frac{(n+p)}{\left[q^{2}+(n+p)^{2}\right]^{2}} \\
& =\frac{\pi^{2}}{q} \frac{\sinh (2 \pi q) \sin (2 \pi p)}{[\cosh (2 \pi q)-\cos (2 \pi p)]^{2}}
\end{aligned}
$$

Integration of eq. (A.2) with respect to $p$ gives

$$
\begin{aligned}
& \sum_{n=-\infty}^{\infty} \ln \left[q^{2}+(n+p)^{2}\right] \\
& \quad=\ln [\cosh (2 \pi q)-\cos (2 \pi p)]
\end{aligned}
$$


while integration of eq. (A.2) over $q$ yields

$$
\begin{aligned}
& \sum_{n=-\infty}^{\infty} \tan ^{-1}\left(\frac{q}{n+p}\right) \\
& =\tan ^{-1}\left(\frac{\sinh (\pi q) \cos (\pi p)}{\cosh (\pi q) \sin (\pi p)}\right) .
\end{aligned}
$$

\section{References}

[1] J.F. van der Veen and M.A. Van Hove, Eds., The Structure of Surfaces II, Vol. 11 of Springer Series in Surfacc Sciences (Springer, Berlin, 1988).

[2] S.M. Yalisove and W.R. Graham, J. Vac. Sci. Technol. 6 (1988) 588.

[3] M.W. Finis and V. Heine, J. Phys. F 4 (1974) L37.

[4] S.P. Chen, A.F. Voter and D.J. Srolovitz, Phys. Rev. Lett. 57 (1986) 1308.

[5] V.I. Marchenko and A.Ya. Purshin, Sov. Phys. JETP 52 (1980) 129.
[6] K.H. Lau and W. Kohn, Surf. Sci. 65 (1977) 607.

[7] V.I. Marchenko, Sov. Phys. JETP Lett. 33 (1981) 381.

[8] V.I. Marchenko, Sov. Phys. JETP Lett. 35 (1982) 567.

[9] O.L. Alerhand, D. Vanderbilt, R.D. Meade and J.D. Joannopoulos, Phys. Rev. Lett. 61 (1988) 1973.

[10] S.P. Chen, A.F. Voter and D.J. Srolovitz, J. Mater. Res. 4 (1989) 64.

[11] A.E.H. Love, The Mathematical Theory of Elasticity (Cambridge University Press, Cambridge, 1927).

[12] M.A. Van Hove, W.H. Weinberg and C.-M. Chan, LowEnergy Diffraction: Experiment, Theory, and Surface Structure (Springer, Berlin, 1986).

[13] R. Feidenhans'l, J.E. Sørensen and I. Stensgaard, Surf. Sci. 134 (1983) 329.

[14] W.K. Burton, N. Cabrera and F.C. Frank, Philos. Trans. R. Soc. (London) A 243 (1951) 299.

[15] A.F. Voter and S.P. Chen, Proc. Mater. Res. Soc. Symp. 82 (1987) 175.

[16] S.P. Chen, unpublished results.

[17] J.P. Hirth and J. Lothe, Theory of Dislocations (Wiley, New York, 1982).

[18] P.M. Morse and H. Feshbach, Methods of Theoretical Physics, Vol. 1 (McGraw-Hill, New York, 1953). 